\title{
Extensor quadriga: Pathomechanics and treatment
}

\author{
Shrikant J Chinchalkar BSCOT OTR CHT ${ }^{1,2}$, Bing Siang Gan MD PhD FRCSC FACS ${ }^{2-6}$, \\ Robert M McFarlane MD FRCSC ${ }^{2,3}$, Graham J King MD FRCSC ${ }^{2,4,6}$, James H Roth MD FRCSC FACS ${ }^{2,4}$
}

SJ Chinchalkar, BS Gan, RM McFarlane, GJ King, JH Roth. Extensor quadriga: Pathomechanics and treatment. Can J Plast Surg 2004;12(4):174-178.

The extensor tendons to the index, long, ring and small fingers are motored by the common extensor digitorum communis muscle body. Effective function of this muscle can only occur if the gliding amplitude of each of its four extensor tendons is normal. As a corollary, limitation of the excursion of any of the individual tendons by adhesions at a fracture or tendon repair site, a fixed flexion contracture at the metacarpophalangeal joint, or by rupture, attenuation or laceration of a saggital band or juncturae tendinum, will result in reduction of the excursion of the adjacent extensor tendons. This pathological state has been termed the extensor quadriga because of its similarities to the analogous pathology affecting the flexor digitorum profundus system. Improper management of this clinical entity may lead to an abnormal pathomechanical kinematic chain imbalance. Early identification and treatment is critical to address this entity appropriately.

Key Words: Extensor lag; Extensor tendon biomechanics; Extensor tendon complications; Extensor quadriga

\section{Syndrome du « quadrige des extenseurs » : pathomécanique et traitement}

Les tendons extenseurs de l'index, du majeur, de l'annulaire et de l'auriculaire sont mus par le muscle extenseur commun des doigts. Mais celuici ne peut fonctionner adéquatement que si l'amplitude de glissement de chacun des quatre tendons extenseurs est normale. En corollaire, une restriction de mouvement de l'un ou l'autre des quatre tendons, due à des adhérences en un foyer de fracture ou au siège de réparation d'un tendon, à une contracture fixe en flexion à une articulation métacarpo-phalangienne ou encore à une rupture, à une faiblesse ou à une lacération d'un ligament sagittal ou du tendon commun, entraîne une restriction de mouvement des tendons extenseurs adjacents. On a donné à ce syndrome le nom de « quadrige des extenseurs » en raison de ses ressemblances avec un syndrome analogue qui touche le muscle fléchisseur commun profond des doigts. Un traitement inapproprié de ce trouble clinique peut conduire à un déséquilibre de la chaîne cinématique pathomécanique. Aussi est-il crucial de reconnaître et de traiter précocement ce syndrome afin d'en assurer une prise en charge adéquate.
$V^{e}$ erdan (1) first reported the syndrome of quadriga in 1960. The term 'quadriga' (Roman four-horsed chariot) was used to illustrate the analogy between the single driver of a Roman chariot holding four reins to four different horses, and the single flexor digitorum profundus muscle (FDP) motoring four individual digital flexor tendons. Like the driver in the quadriga, the common FDP muscle body limits independent function of the flexor tendons. Verdan further outlined that the FDP muscle body can only contract effectively if the excursion of each of the flexor tendons is normal. Thus, if one of the tendons is fixed, its reduced gliding excursion will result in decreased amplitude of the muscle, which in turn will have an effect on adjacent tendons (1). The gliding amplitude of the flexor tendons can also be affected by proximal interphalangeal (PIP) or distal interphalangeal (DIP) joint flexion contractures, producing a pseudoquadriga effect. Verdan correctly concluded that in such instances, the fixed tendon would have to be released to restore normal excursion to the other tendons and achieve normal hand function.

It is less well recognized that a similar arrangement exists on the extensor surface of the hand, with a single extensor digitorum communis (EDC) muscle motoring four extensor tendons. Thus, an analogous extensor quadriga can be present if the gliding amplitude of one of the individual extensor tendons is reduced, such as in flexion contractures of the metacarpophalangeal (MCP) joint or adhesion formation following fractures in the phalanges or metacarpals. Unfortunately, this syndrome is often not recognized, leading to inappropriate therapeutic approaches. In the present article, we will discuss the pathomechanics of extensor quadriga.

\section{BIOMECHANICS OF NORMAL DIGITAL EXTENSION}

To appreciate and recognize the pathomechanics of extensor quadriga, an awareness of extensor anatomy and the mechanism of normal digital flexion and extension are critical. Despite some anatomical variations, the extensor anatomy proximal to the sagittal bands is relatively simple when compared with the distal extensor system (2). The four EDC tendons to the index, long, ring and small fingers emerge from the fourth extensor compartment at the wrist and diverge as they advance toward their insertion. These extensor tendons are

\footnotetext{
${ }^{1}$ Department of Hand Therapy and ${ }^{2}$ Hand and Upper Limb Centre, St Joseph's Health Care London; Divisions of ${ }^{3}$ Plastic and ${ }^{4}$ Orthopedic Surgery, Department of Surgery; Divisions of 5 Physiology and Pharmacology and ${ }^{6}$ Medical Biophysics, University of Western Ontario, London, Ontario
}

Correspondence and reprints: Shrikant J Chinchalkar, Department of Hand Therapy, Hand and Upper Limb Centre, 268 Grosvenor Street, London, Ontario N6A 4L6. Telephone 519-646-6000 ext 65506, fax 519-646-6049, e-mail Shrikant.Chinchalkar@sjhc.london.on.ca 


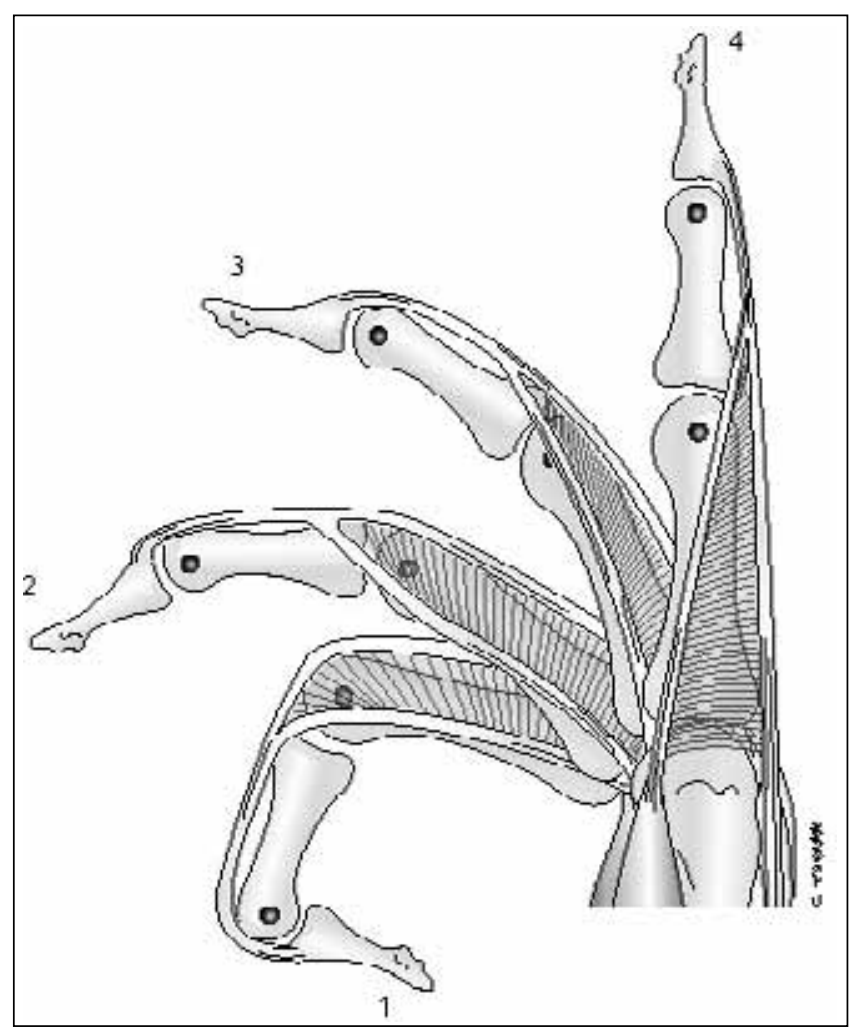

Figure 1) Sequential stages of digital extensor mechanism. The extensor digitorum communis (EDC) central slip initiates proximal interphalangeal joint extension (1), resulting in dorsal migration of the lateral bands. This leads to extension of the distal interphalangeal joint (2). The intrinsic muscles contribute to further extension of the distal interphalangeal and proximal interphalangeal joints by adding tension to the lateral bands, while the EDC continues to glide proximally (3). Continued proximal migration of the EDC tendon leads to tightening of the saggital bands, which act as a sling around the base of the proximal phalanx, thus leading to metacarpophalangeal extension (4)

interconnected by the juncturae tendinum and the intertendinous fascia. The juncturae tendinum play an important role in maintaining the divergent angle and, at the same time, supplement transmission of extensor force during digital extension (3). It is suspected that these structures provide some stability to the sagittal bands $(3,4)$. Distal to the juncturae tendinum, the sagittal bands stabilize the extensor tendons over the $\mathrm{MCP}$ joint. In addition to providing stability to the MCP joint in flexion, the sagittal bands are dynamic structures that move with the extensor tendon during MCP joint motion. Together with the volar plate, they work as a sling around the base of the proximal phalanx and assist the EDC tendon in extending the MCP joint $(5,6)$.

In free hand motion, from a fully flexed digital position, the central slip of the EDC tendon first initiates PIP joint extension. As the PIP joint gains some extension, the conjoined lateral bands gradually migrate dorsally, causing tension at the DIP joint. As a result, DIP joint extension is initiated. The intrinsic muscles, through the medial bands and the conjoined lateral bands acting at the PIP and the DIP joints, continue to extend these joints, respectively. As the PIP and DIP joints continue to extend, the EDC continues to pull the sagittal



Figure 2) Simulated extensor digitorum communis (EDC) adhesion at the level of the metacarpal neck. A K-wire is driven through the EDC tendon at the level of the metacarpal neck. This leads to a reduced gliding amplitude of the EDC tendon in the involved digit and, thus, a decrease in extension of particularly the metacarpophalangeal joint. Extension of the proximal interphalangeal and distal interphalangeal joints may be maintained through the action of the intrinsic mechanism

bands proximally, bringing the sagittal bands over the metacarpal head and acting as a sling on the proximal phalanx, simultaneously extending the MCP joint (7). The juncturae tendinum maintain the extensor force while stabilizing the extensor tendons over the dorsum of the MCP joints with the assistance of the sagittal bands. The oblique force of the intrinsic muscles and the linear force of the EDC maintain complete digital extension at this stage (Figure 1) $(3,6)$.

\section{BIOMECHANICS OF EXTENSOR QUADRIGA}

Reduced gliding amplitude of the extensor tendon is commonly seen in conditions such as adhesion formation following extensor tendon repair, metacarpal fractures (8), sagittal band attenuation or ruptures $(5,9)$, ruptures or attenuation of juncturae tendinum (10) and flexion contractures of the MCP joint. The pathomechanics of extensor quadriga in each of these conditions is described below.

\section{Extensor quadriga in metacarpal fractures and extensor} tendon repairs at zones $\mathrm{V}$ and VI

EDC tendon adhesions following metacarpal fractures and extensor tendon repairs in zones $\mathrm{V}$ and $\mathrm{VI}$ and proximally are a common complication $(11,12)$. The adhesion of the extensor tendon causes a pseudoinsertion at the tethered site (Figures 2 and 3). The tethered tendon of the affected digit produces an extensor lag at the MCP joint of the involved ray because of the reduced gliding amplitude of the EDC tendon distal to the adhesion (Figure 3). In addition, if the fourth or fifth ray is involved, tendon adhesions may produce abnormal motion at 


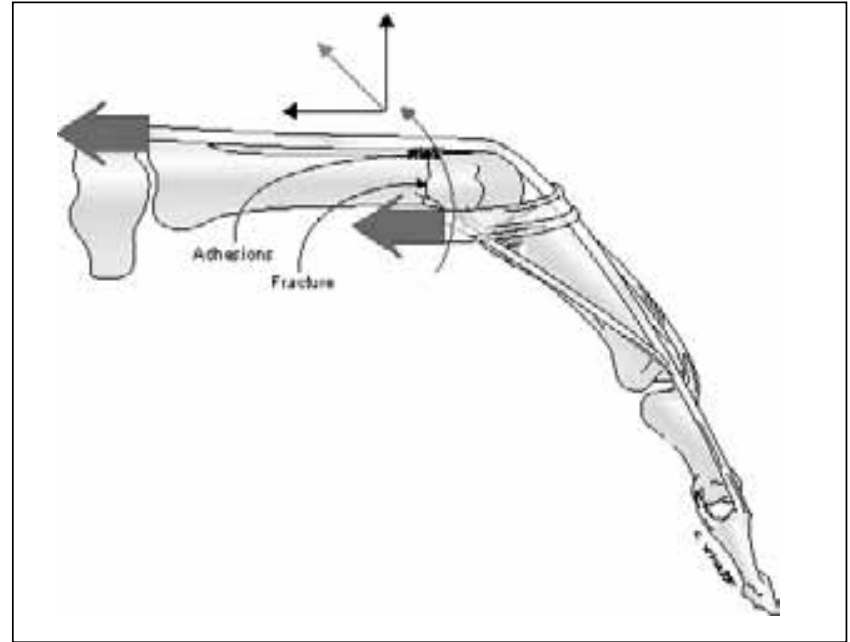

Figure 3) Extensor digitorum communis (EDC) adhesions in zones $\mathrm{V}$ and VI. If the EDC tendon develops reduction of gliding amplitude in zones $V$ or VI as a result of tendon repair or metacarpal fracture, the involved digit exhibits reduced extension of particularly the metacarpophalangeal joint (see Figure 1, stage 4). The proximal interphalangeal and distal interphalangeal extension may be maintained through intrinsic forces. Because the adhesions now lead to EDC force transmission to the distal part of the involved metacarpal, this may result in carpometacarpal hyperextension. This is more common if the fourth and fifth rays are involved

the carpometacarpal joints during extension (Figures 3 and 4). The EDC tendon adhesion at the metacarpal site causes hyperextension at the hamatometacarpal joint of these digits. This hyperextension motion at this joint may also reduce the tension on adjacent EDC tendons, further contributing to the quadriga effect (Figure 4).

Extensor quadriga in sagittal band and juncturae tendinum rupture, laceration or attenuation

The sagittal bands and juncturae tendinae transmit precise forces during the flexion and extension of the digits. The sagittal bands are part of the complex extensor retinacular system. They surround the metacarpal head and the MCP joint with an attachment at the volar plate (13). The sagittal bands, in collaboration with the EDC tendons, extend the MCP joint. Extensor tendon instability over the MCP joint results from failure of the sagittal bands (Figure 5). The radial segment of the sagittal bands is the most vulnerable to stress, and attenuation causes ulnar subluxation of the extensor tendon (5). As a result, upon EDC activation for digital extension, the sagittal bands fail to extend the MCP joint in phase 4 of digital extension (Figure 1). The lack of extension of the involved digit subsequently results in a decreased excursion of the adjacent EDC tendons through distal tension on the juncturae tendinum. A similar deficiency of the sagittal band can be seen in the rheumatoid hand, also producing a extensor quadriga phenomenon.

Extensor quadriga caused by MCP joint flexion contracture Distal limitation of the EDC tendon excursion caused by DIP, PIP or MCP joint contractures reduce the gliding amplitude of these extensor tendons proximally. This deficiency of the

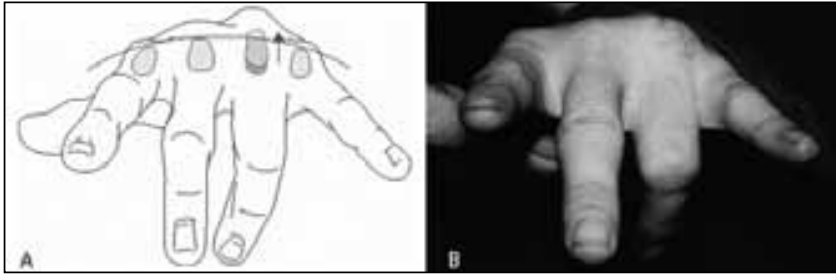

Figure 4) Carpometacarpal (CMC) hyperextension contributes to the quadriga effect. Extensor digitorum communis adhesions in zones $V$ and VI may lead to abnormal CMC hyperextension (A). This is most pronounced in the fourth and fifth rays. CMC hyperextension leads to reduction in tension of the distal extensor digitorum communis complex in the adjacent digits, contributing to a decreased gliding amplitude in these digits and, thus, a quadriga effect (B)

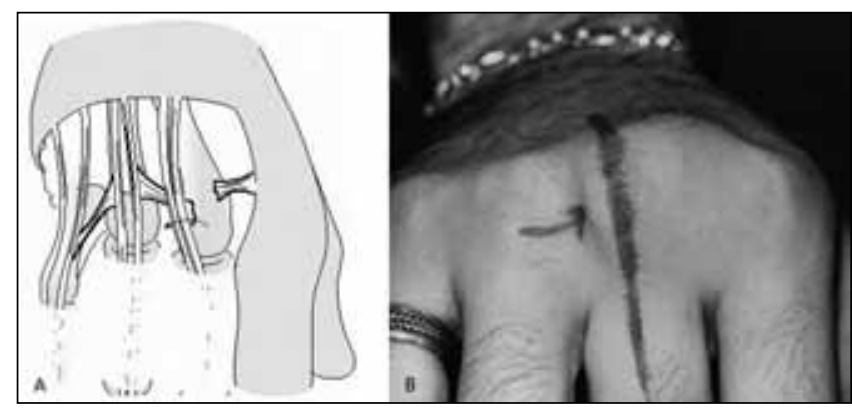

Figure 5) A Rupture of the saggital bands decreased effective amplitude of extensor digitorum communis (EDC) tendon. Rupture of the saggital band mechanism around the metacarpophalangeal joint occurs most commonly on the radial side. This results in subluxation of the EDC tendon into the valleys between the heads of the metacarpals. This leads to a decreased effective excursion of the EDC tendon and an extensor lag, mostly at the metacarpophalangeal joint. In panel $\mathbf{B}$, the normal tract of the EDC tendon is marked and the arrow indicates the subluxed EDC tendon

extensor tendon gliding proximally, due to a contracture especially at the MCP joint of an involved digit, will result in a quadriga effect on the adjacent digits. This has been observed in flexion contractures and Dupuytren's contractures affecting the MCP joint. The quadriga effect of distal joint contractures is most pronounced when this occurs in the third ray. This is because of the orientation of the juncturae tendinum, which are located from proximal in the index ray to distal in the long finger ray and from proximal in the ring ray to distal in the long ray. This orientation firmly prevents further proximal excursion of the ring and index EDC beyond that which is allowed by the long finger EDC.

\section{COMPLICATIONS OF EXTENSOR QUADRIGA}

The quadriga effect mainly results in a decrease in extension of the MCP joints. As a result, the intrinsic muscles remain in a shortened position and, over time, can develop intrinsic tightness. To compensate for loss of extension at the MCP joint, the intrinsic muscles contribute an excessive extensor load at the PIP joint. When myostatic contracture of intrinsic structures occurs, the result is a secondary swan neck deformity. Over time, the digits will demonstrate intrinsic tightness and secondary 

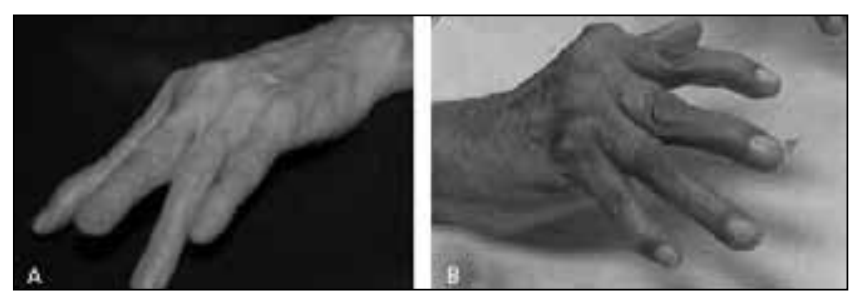

Figure 6) Swan neck deformity resulting from extensor quadriga. Saggital band rupture in the ring finger of this patient resulted in extension deficit of this digit and a quadriga effect on all adjacent digits. Note the swan neck deformity in the long and index fingers (A). Ulnar drift and sagittal band attenuation with volar subluxation of the metacarpophalangeal joints in this rheumatoid patient led to extensor quadriga and marked swan neck deformity (B)

swan neck tendency if the pathomechanics are not addressed early (Figure 6). This complication is particularly clear in the rheumatoid hand, where attenuation of the sagittal bands of one digit may lead to extensor quadriga and, over time, the development of swan neck deformities of all digits (Figure 6). In addition, in rheumatoid swan neck deformities, continuous load of the intrinsics at the proximal phalanx (through the transverse retinacular fibers of the intrinsics) may be one of the main causes of volar subluxation and ulnar deviation of the MCP joint (14). Finally, in extreme extensor quadriga cases, after the development of a swan neck deformity, a reversal flexor quadriga may result.

\section{TREATMENT}

Early treatment of this syndrome is based on the recognition of the primary pathology. Early mobilization of digits with a metacarpal brace in metacarpal fractures treated with open reduction and internal fixation facilitates gliding of the extensor tendons; this reduces the possibility of a quadriga effect. The purpose of the metacarpal brace is to position the distal metacarpal arch and, at the same time, reduce the carpometacarpal extension if the adhesions are forming at the fracture site. Fractures treated with closed reduction in an intrinsic plus splint or cast are likely to produce some degree of intrinsic tightness because of the positioning of the digits. This intrinsic tightness is then compounded by adhesion formation at the fracture site, entrapping the EDC tendon. It is, therefore, important to recognize that after confirmation of fracture healing, intrinsic stretching exercises should be added to therapy aimed at restoring EDC excursion.

An effective way to maintain EDC extensor tendon excursion after repair of zones V and VI is the reverse Kleinert technique (15). Tendon adhesions are minimized and the extensor quadriga effect is rarely observed. Extensor tendon adhesions following a traditional treatment of tendon immobilization with a splint may result in an extensor lag. To minimize the chances of developing extensor quadriga, modalities such as ultrasound and electrical stimulation may be required to enhance tendon gliding. The excursion of the extensor tendon can be maximized by continuous use of a figure-of-eight PIP splint until tendon excursion is restored. A resisted extension exercise program further enhances tendon excursion. Tendons failing to gain excursion proximally due to excessive firm adhesion formation may benefit by early tenolysis and an immediate mobilization program. If early tenolysis is not possible, the prevention of PIP joint hyperextension and formation of intrinsic tightness should be next in line as primary therapeutic goals.

Traumatic sagittal band and/or juncturae tendinum tears and/or ruptures in the nonrheumatoid hand can be successfully treated with triple buddy taping techniques. If extensor tendon instability persists and restoration of sagittal band stability is not successful using closed means, a surgical repair along with controlled arc motion program with the reverse Kleinert technique will produce favourable results.

Attenuation of the sagittal bands in rheumatoid hand is one of the causes of ulnar subluxation of the EDC tendon. The subluxation of one EDC tendon leading to subsequent subluxation of adjacent tendons and extensor quadriga can be minimized with early detection and surgical reconstruction of the sagittal band and centralization of the EDC. Following surgical reconstruction, the repaired digit can be successfully treated with the reverse Kleinert technique. With early surgical management of the sagittal bands, secondary complications such as the development of swan neck deformities and volar subluxation of the MCP joints can be prevented, thus preserving MCP joint stability.

Flexion contractures at the MCP joints, other than those caused by Dupuytren's contracture, may be treated successfully with dynamic or static progressive serial splints or a combination of these methods depending on the end feel of the restraining tissues. In particular, adjacent digits with a tendency for intrinsic tightness and/or swan neck deformity must be vigilantly treated.

In patients with established extensor quadriga associated with swan neck deformity, a critical and indepth assessment of the contributing factors is paramount to planning a surgical procedure. Before an operative procedure, a well-structured therapy program consisting of intrinsic stretching, splinting to prevent swan neck tendency and maximizing EDC excursion will reduce the associated contributing factors and make the surgical procedure simpler. A carefully planned therapy program would be required based on the surgical procedure to maximize the outcome.

\section{CONCLUSION}

The syndrome of extensor quadriga can occur when the gliding amplitude of the EDC tendon of any digit is limited. This reduced gliding amplitude has a significant effect on the adjacent EDC tendon excursion, and will result in an extension deficit of adjacent digits. Reduced EDC gliding amplitude can occur after sagittal band or juncturae tendinum rupture or attenuation, EDC tendon tethering after a metacarpal fracture, zones $\mathrm{V}$ and VI or proximal extensor tendon repair, flexion contracture affecting the MCP joint or any other pathology limiting the excursion of an EDC tendon. The complications of this entity, in particular intrinsic tightness, can be serious and, therefore, early detection and management of this condition are critical in preserving hand function.

\section{REFERENCES}

1. Verdan C. Syndrome of the quadriga. Surg Clin North Am 1960;40:425-6.

2. von Schroeder HP, Botte MJ. Anatomy of the extensor tendons of the fingers: Variations and multiplicity. J Hand Surg [Am] 1995;20:27-34. 
3. von Schroeder HP, Botte MJ. The functional significance of the long extensors and juncturae tendinum in finger extension. J Hand Surg [Am] 1993;18:641-7.

4. von Schroeder HP, Botte MJ, Gellman H. Anatomy of the juncturae tendinum of the hand. J Hand Surg [Am] 1990;15:595-602.

5. Young CM, Rayan GM. The sagittal band: Anatomic and biomechanical study. J Hand Surg [Am] 2000;25:1107-13.

6. Gausepohl T, Koebke J, Pennig D, Thiel J. Changes in the form of the interosseous hood during extension and flexion of the metacarpophalangeal joint. Handchir Mikrochir Plast Chir 1998;30:220-5.

7. Valentin P. Physiology of extension of fingers. In: Tubiana R, ed. The Hand. Philadelphia: WB Saunders, 1981:389-98.

8. Strauch RJ, Rosenwasser MP, Lunt JG. Metacarpal shaft fractures: The effect of shortening on the extensor tendon mechanism. J Hand Surg [Am] 1998;23:519-23.

9. Wilhelm A. The quadriga phenomenon of the extensor tendon system and the superficial transverse metacarpal ligament. Handchir Mikrochir Plast Chir 1988;20:173-9. German.

10. von Schroeder HP, Botte MJ. Functional anatomy of the extensor tendons of the digits. Hand Clin 1997;13:51-62.

11. Green DP. Complications of phalangeal and metacarpal fractures. Hand Clin 1986;2:307-28.

12. Dovelle S, Heeter PK, Fischer DR, Chow JA. Rehabilitation of extensor tendon injury of the hand by means of early controlled motion. Am J Occup Ther 1989;43:115-9.

13. Rayan GM, Murray D, Chung KW, Rohrer M. The extensor retinacular system at the metacarpophalangeal joint. Anatomical and histological study. J Hand Surg [Br] 1997;22:585-90.

14. Wilkes LL. Ulnar drift and metacarpophalangeal joint subluxation in the rheumatoid hand: Review of the pathogenesis. South Med J 1977;70:963-7.

15. Evans RB. Clinical management of extensor tendons. In: Mackin EJ, Skirven TM, Schneider LH, Ostermen AL, eds. Rehabilitation of the Hand and Upper Extremity. St Louis: Mosby, 2002:542-79. 